Recepción: 22 / 10 / 2017

Aceptación: 27 / $11 / 2017$

Publicación: 15/ 12/2017

Ciencias naturales

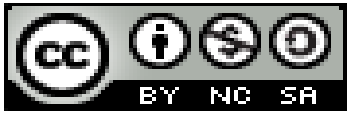

Artículo de investigación

\title{
Principales características de las plantas de cogeneración
}

Main characteristics of cogeneration plants

Principais características das plantas de cogeração

Franklin M. Reina-Quiñónez ${ }^{\mathrm{I}}$

ingfranklinreynaq@hotmail.com

Paul R. Vizcaíno-Valencia ${ }^{\text {II }}$ pavisva@hotmail.com

Mirtha L. Aguilar-Mora ${ }^{\mathrm{III}}$ lore322@hotmail.es

Alvez R. Mera-Mosquera ${ }^{\text {Iv }}$ meraromel@hotmail.es

Correspondencia: ingfranklinreynaq@hotmail.com

I. Master en Ciencias Especialidad Docencia Universitaria, Ingeniero Industrial, Docente de la Universidad Técnica Luis Vargas Torres de Esmeraldas, Esmeraldas, Ecuador.

II. Ingeniero Mecánico, Docente de la Universidad Técnica Luis Vargas Torres de Esmeraldas, Esmeraldas, Ecuador.

III. Ingeniera Mecánica, Docente de la Universidad Técnica Luis Vargas Torres de Esmeraldas, Esmeraldas, Ecuador.

IV. Ingeniero en Mecánica Automotriz, Tecnólogo en Mecánica Automotriz: Mantenimiento y Reparación de Motores a Diesel y Gasolina, Docente de la Universidad Técnica Luis Vargas Torres de Esmeraldas, Esmeraldas, Ecuador. 


\section{Resumen}

El siguiente trabajo aborda las características fundamentales de las plantas de cogeneración en los procesos industriales. Para ello, presenta las conceptualizaciones de los términos vinculados al tema, de igual forma, describe el empleo de dichas plantas mediante la explicación de sus elementos estructurales, sus principales tipos y las diversas configuraciones posibles. Todo ello se realiza a través de diferentes métodos de investigación teóricos.

Palabras clave: plantas de cogeneración; procesos industriales; turbinas de gas; motor de gas; turbinas de vapor.

\section{Abstract}

The following work addresses the fundamental characteristics of cogeneration plants in industrial processes. For this, it presents the conceptualizations of the terms linked to the subject, likewise, it describes the use of said plants by explaining their structural elements, their main types and the different possible configurations. All this is done through different theoretical research methods. Keywords: cogeneration plants; industrial processes; gas turbines; gas engine; steam turbines.

\section{Resumo}

O trabalho a seguir aborda as características fundamentais das plantas de cogeração em processos industriais. Para isso, apresenta as conceituações dos termos ligados ao sujeito, da mesma forma, descreve o uso das referidas plantas, explicando seus elementos estruturais, seus principais tipos e as diferentes configurações possíveis. Tudo isso é feito através de diferentes métodos de pesquisa teórica.

Palavras chave: plantas de cogeração; processos industriais; turbinas a gás; motor a gás; turbinas a vapor.

\section{Introducción}

Los debates sobre los temas medioambientales en la Organización de Naciones Unidas y fundamentalmente en una de sus agencias especializadas, el Programa de las Naciones Unidas para el Medio Ambiente (UNEP), cobran cada día mayor auge. 
¿Cómo utilizar la energía de forma eficiente? ¿Cómo aprovechar todos los recursos energéticos sin dañar la capa de ozono? La pregunta carece de una respuesta exacta, pero sí de ciertas variantes posibles.

En las industrias, son innumerables los procesos químicos y físicos que tienen lugar, lo que lleva consigo alteraciones medioambientales. Sin embargo, se han creado alternativas para que estas alteraciones sean mínimas, una de ellas son las plantas de cogeneración.

"El término cogeneración se emplea para designar la producción secuencial de energía térmica y energía eléctrica a partir de una fuente primaria de energía, estando normalmente esta producción de energía (térmica y eléctrica) ligada a un proceso Industrial, Comercial o de Servicios. Las plantas de Cogeneración producen, pues, de forma simultánea electricidad y calor para diversas aplicaciones. El atractivo fundamental de este tipo de plantas se basa en que tienen una óptima eficiencia en las transformaciones energéticas, con mínimos consumos de combustible primario y con un menor impacto ambiental". (Energiza, s/f)

"La cogeneración se define como la producción simultánea de energía mecánica transformada en electricidad y energía térmica útil. Este proceso es eficiente porque contribuye al ahorro energético y disminuye los niveles de contaminación, convirtiéndose en una efectiva herramienta para la lucha contra el cambio climático. Se sabe que la termodinámica obliga a la evacuación de una cierta cantidad de calor en todo proceso térmico de producción de electricidad, ya que todo el calor absorbido no puede transformarse en trabajo, y el objetivo de la cogeneración es que no se pierda esta gran cantidad de energía. Esta producción simultánea supone que puede ser utilizada simultáneamente, lo que implica proximidad de la planta generadora a los consumos, al contrario del sistema convencional de producción de electricidad en centrales termoeléctricas independientes, donde también se desprende calor, pero este no es aprovechado $\mathrm{y}$ ha de ser eliminado al ambiente". (Khellaf, 2016)

Si bien toda planta que utilice y transforme energía le resulta imposible no dañar el medio ambiente, el ser humano de cierta forma ha intentado crear vías y medios para afectar lo menos posible la capa de ozono, las plantas de cogeneración son un ejemplo de ello. "El atractivo fundamental de este tipo de plantas se basa en que tienen una óptima eficiencia en las 
transformaciones energéticas, con mínimos consumos de combustible primario y con un menor impacto ambiental". (Energiza, s/f)

Este trabajo se propone como objetivo general:

- Describir las principales características de las plantas de cogeneración.

Para ello, se abordarán 4 interrogantes fundamentalmente:

- ¿Qué son las plantas de cogeneración?

- ¿Cuáles son los principales tipos de plantas de cogeneración?

- ¿Cuáles son los elementos comunes a todas las plantas de cogeneración?

- ¿Cuáles son las configuraciones posibles para las plantas de cogeneración?

\section{Metodología}

Para cumplir con ello, se utilizan diferentes métodos teóricos:

- Revisión bibliográfica a través de diferentes palabras claves, estrategias de búsquedas, recursos de información.

- Análisis-Síntesis

- Inducción-Deducción

- Hipotético deductivo

- Histórico y lógico

\section{Desarrollo}

Según Santiago García Garrido, la cogeneración significa "producción simultánea de dos o más tipos de energía. Normalmente las energías generadas son electricidad y calor, aunque puede ser también energía mecánica y calor (y/o frío). La producción simultánea supone que puede ser utilizada simultáneamente, lo que implica proximidad de la planta generadora a los consumos, en contraposición al sistema convencional de producción de electricidad en centrales termoeléctricas 
independientes, donde también se desprende calor, pero éste no es aprovechado y ha de ser eliminado al ambiente". (García, s/f)

Las normas generales de la termodinámica exigen el desecho de una cierta cantidad de energía que se transforma en calor, pues no toda se utiliza en los procesos. Precisamente el objetivo de las plantas de cogeneración es aprovechar esa energía que se desprende.

En la literatura revisada aparecen los diferentes tipos de plantas de cogeneración: (Renove Tecnología S.L, s/f)

\section{- COGENERACIÓN CON MOTOR DE GAS}

Utilizan gas, gasóleo o fuel-oil como combustible. Son muy eficientes eléctricamente, pero son poco eficientes térmicamente. El sistema de recuperación térmica se diseña en función de los requisitos de la industria y en general se basan en la producción de vapor a baja presión (hasta 10 bares), aceite térmico y en el aprovechamiento del circuito de alta temperatura del agua de refrigeración del motor. Son también adecuadas la producción de frío por absorción, bien a través del vapor generado con los gases en máquinas de doble efecto, o utilizando directamente el calor del agua de refrigeración en máquinas de simple efecto.

Este tipo de instalaciones es conveniente para potencias bajas (hasta $15 \mathrm{MW}$ ) en las que la generación eléctrica es muy importante en el peso del plan de negocio. Los motores son la máquina térmica que más rendimiento tiene, pues es capaz de convertir actualmente hasta el 45\% de la energía química contenida en el combustible en energía eléctrica, y se espera que en los próximos años este rendimiento aumente.

\section{- COGENERACIÓN CON TURBINA DE GAS}

En los sistemas con turbina de gas se quema combustible en un turbogenerador. Parte de la energía se transforma en energía mecánica, que se transformará con la ayuda del alternador en energía eléctrica. Su rendimiento eléctrico es inferior al de los motores alternativos, pero presentan la ventaja de que permiten una recuperación fácil del calor, que se encuentra 
concentrado en su práctica totalidad en sus gases de escape, que está a una temperatura de unos $500^{\circ} \mathrm{C}$, idónea para producir vapor en una caldea de recuperación

Cuando se presenta en el denominado ciclo simple, el sistema consta de una turbina de gas y una caldera de recuperación, generándose vapor directamente a la presión de utilización en la planta de proceso asociada a la cogeneración. Su aplicación es adecuada cuando los requisitos de vapor son importantes $(>10 \mathrm{t} / \mathrm{h})$, situación que se encuentra fácilmente en numerosas industrias (alimentación, química, papelera). Son plantas de gran fiabilidad y económicamente rentables cuando están diseñadas para una aplicación determinada.

El diseño del sistema de recuperación de calor es fundamental, pues su economía está directamente ligada al mismo, ya que a diferencia de las plantas con motores alternativos el precio del calor recuperado es esencial en un ciclo simple de turbina de gas.

\section{- COGENERACIÓN CON TURBINA DE VAPOR}

En estos sistemas, la energía mecánica se produce por la expansión del vapor de alta presión procedente de una caldera convencional. El uso de esta turbina fue el primero en cogeneración. Actualmente su aplicación ha quedado prácticamente limitada como complemento para ciclos combinados o en instalaciones que utilizan combustibles residuales, como biomasa subproductos residuales que se generan en la industria principal a la que está asociada la planta de cogeneración.

Dependiendo de la presión de salida del vapor de la turbina se clasifican en turbinas a contrapresión, en donde esta presión está por encima de la atmosférica, y las turbinas a condensación, en las cuales ésta está por debajo de la atmosférica y han de estar provistas de un condensador. En ambos casos se puede disponer de salidas intermedias, extracciones, haciendo posible la utilización en proceso a diferentes niveles de presión

\section{- COGENERACIÓN EN CICLO COMBINADO CON TURBINA DE GAS Y VAPOR}

La aplicación conjunta de una turbina de gas y una turbina de vapor es lo que se denomina " Ciclo Combinado". 
En el gráfico adjunto puede verse que los gases de escape de la turbina pueden tirarse a la atmósfera si no se requiere aprovechamiento térmico, a través del bypass, o pueden atravesar la caldera de recuperación, donde se produce vapor de alta presión. Este vapor puede descomprimirse en una turbina de vapor produciendo una energía eléctrica adicional. La salida de la turbina será vapor de baja presión, que puede aprovecharse como tal o condensarse en un condensador presurizado, produciendo agua caliente o agua sobrecalentada, que será utilizado en la industria asociada. Si la demanda de vapor es mayor que la que pueden proporcionar los gases de escape, puede producirse una cantidad de vapor adicional utilizando un quemador de postcombustión, introduciendo una cantidad adicional de combustible (gas natural) directamente a un quemador especial con el que cuenta la caldera. Esto puede hacerse porque los gases de escape son aun suficientemente ricos en oxígeno (en un ciclo combinado con motor alternativo no podría hacerse, ya que los gases de escape son pobres en oxígeno).

En un ciclo combinado con turbina de gas el proceso de vapor es esencial para lograr la eficiencia del mismo. La selección de la presión y la temperatura del vapor vivo se hace en función de las turbinas de gas y vapor seleccionadas, selección que debe realizarse con criterios de eficiencia y economía. Por ello se requiere una ingeniería apropiada capaz de crear procesos adaptados al consumo de la planta industrial asociada a la cogeneración, que al mismo tiempo dispongan de gran flexibilidad que posibilite su trabajo eficiente en situaciones alejadas del punto de diseño.

Una variante del ciclo combinado expuesto, en el que la turbina de vapor trabaja a contrapresión (esto es, descomprime el vapor entre una presión elevada y una presión inferior, siempre superior a la atmosférica) es el ciclo combinado a condensación, en el que el aprovechamiento del calor se realiza antes de la turbina de vapor, quedando ésta como elemento final del proceso. El vapor de salida se condensa en un condensador que trabaja a presión inferior a la atmosférica, para que el salto térmico sea el mayor posible.

\section{- COGENERACIÓN CON MOTOR DE GAS Y TURBINA DE VAPOR}

En este tipo de plantas, el calor contenido en los humos de escape del motor se recupera en una caldera de recuperación, produciendo vapor que es utilizado en una turbina de vapor para producir más energía eléctrica o energía mecánica. El circuito de refrigeración de alta 
temperatura del motor se recupera en intercambiadores, y el calor recuperado se utiliza directamente en la industria asociada a la planta de cogeneración. El rendimiento eléctrico en esta planta es alto, mientras que el térmico disminuye considerablemente."

A partir de las fuentes de información revisadas se puede decir que los elementos comunes en una planta de cogeneración son: (Energiza, s/f)

Fuente de energía primaria. Suele ser gas natural, gasoil o fuel oil.

1. El elemento motor. Es el elemento encargado de convertir energía térmica en mecánica. Dependiendo del tipo de planta, puede tratarse de turbinas de gas, turbinas de vapor o motores alternativos.

2. El sistema de aprovechamiento de energía mecánica. En general suele estar formado por alternador y transformadores de tensión, pero también puede tratarse de compresores, bombas, etc, donde la energía mecánica se aprovecha directamente.

3. El sistema de aprovechamiento de calor. Puede tratarse de calderas recuperadoras de calor en gases de escape, calderas convencionales, secaderos o intercambiadores de calor.

4. Sistemas de refrigeración. Una parte de la energía térmica contenida en el combustible no será aprovechada en la planta y debe ser evacuada. Las torres de refrigeración. Los aerocondensadores o los intercambiadores suelen ser elementos habituales de estos sistemas.

5. Sistema de tratamiento de agua. Tanto el sistema de refrigeración como el de aprovechamiento de calor requieren unas especificaciones en las características fisicoquímicas del fluido que utilizan (generalmente agua) que requiere de una serie de sistemas para su tratamiento y control.

6. Sistema de control, que se encarga del gobierno de las instalaciones, normalmente muy automatizadas.

7. Sistema eléctrico, que permite tanto la alimentación de los equipos auxiliares de la planta, como la exportación/importación de energía eléctrica necesaria para cumplir el balance. 
La fiabilidad de esta instalación es muy importante, así como la posibilidad de trabajo en isla, lo que permite alimentar la fábrica en situación de deficiencia de la red externa y estar disponible inmediatamente en el momento que se restablezcan las condiciones del servicio".

Asimismo, Khellaf (2016) plantea los siguientes elementos principales de un sistema de cogeneración:

- Turbinas de vapor, Turbinas de gas y Motores de combustión interna. Este se encarga de convertir la energía del combustible en energía mecánica, que es aprovechada para generar energía eléctrica.

- Caldera de recuperación de calor. Su función es recuperar el calor de los gases calientes de la combustión en la turbina de gas, para producir vapor o fluidos calientes.

- Sistemas auxiliares: Bombas, Compresores, Tratamientos de Agua, etc. Estos son los equipos secundarios con los que debe contar el sistema de cogeneración para su óptimo funcionamiento.

- Sistema de control: Necesario para la automatización de la operación del sistema de cogeneración optimizándolo y dándole seguridad.

Las configuraciones posibles para las plantas de cogeneración son:

1. Cogeneración superior: Es aquella en la que una fuente de energía primaria (como: gas natural, diésel, carbón, biomasa, biogás u otro combustible similar) se utiliza directamente para la cogeneración de energía (primer escalón). A partir de la energía química del combustible se genera la energía mecánica que se transforma en electricidad. La energía térmica residual del equipo, como vapor, gases calientes o agua caliente, es suministrada a los procesos industriales ya sea para secado, cocimiento o calentamiento (segundo escalón). Este tipo de cogeneración es el más empleado.

2. Cogeneración inferior: La energía primaria se utiliza directamente para satisfacer los requerimientos térmicos del proceso del primer escalón y la energía térmica residual o de 
desecho, se usará para la generación de energía eléctrica en el segundo escalón. Los ciclos inferiores están asociados con procesos industriales en los que se presentan altas temperaturas (Ministerio de Electricidad y Energía Renovable, s/f) (Ministerio de Electricidad y Energía Renovable, s/f)

\section{Conclusiones}

Después de haber caracterizado las plantas de cogeneración a partir de diferentes aspectos es indispensable enfatizar en las ventajas de las mismas y como Ecuador no se queda afuera de ellas.

El proyecto de San Carlos se convirtió en el primer plan ecuatoriano de cogeneración eléctrica a partir de biomasa en lograr un registro internacional en la Organización de las Naciones Unidas (ONU) como proyecto de Desarrollo Limpio MDL. Desde el 2012, como parte de su compromiso con el medioambiente, están en marcha otros tres programas:

Agricultura de precisión: consiste en la optimización del uso del suelo mediante un manejo diferenciado de los tipos de suelo.

Optimización del Uso del Agua: mediante la mejora en los procesos, se logró disminuir el consumo del agua.

Recuperación de Aceite Usado: creación de una planta destinada al reciclaje y re-uso del aceite usado en las maquinarias y vehículos.

\section{Referencias Bibliográficas}

Energiza. (s/f). Las Plantas de Cogeneración. Recuperado el 02 de febrero de 2017, de http://www.energiza.org/puesta-en-marcha-de-instalaciones/36-las-plantas-de-cogeneracion

García, S. (s/f). Las plantas de cogeneración. Recuperado el 02 de febrero de 2017, de http://www.energia.renovetec.com/centrales-de-ciclo-combinado/298-plantas-de-cogeneracion

Khellaf, S. (2016). Modelado y simulación de una planta de cogeneración mediante el simulador de procesos HYSYS. Sevilla: Proyecto de Fin de Carrera. 
Ministerio de Electricidad y Energía Renovable. (s/f). Cogeneración. Recuperado el 02 de febrero de 2017, de http://www.energia.gob.ec/coogeneracion/

Renove Tecnología S.L. (s/f). Plantas de cogeneración. Recuperado el 02 de febrero de 2017, de http://www.plantasdecogeneracion.com/index.php/las-plantas-de-cogeneracion 